\title{
Response to the Comment on "Bariatric Surgery can Lead to Net Cost Savings to Health Care Systems: Results from a Comprehensive European Decision Analytic Model"
}

\author{
Oleg Borisenko ${ }^{1}$ - Daniel Adam ${ }^{1}$ - Peter Funch-Jensen ${ }^{2,3}$ • Ahmed R. Ahmed ${ }^{4}$. \\ Rongrong Zhang ${ }^{1} \cdot$ Zeynep Colpan $^{1} \cdot$ Jan Hedenbro ${ }^{5,6}$
}

Published online: 1 May 2015

(C) The Author(s) 2015. This article is published with open access at Springerlink.com

Dear Editor,

We are grateful for having the possibility to comment on the "Letter to the editor" regarding our article "Bariatric Surgery can Lead to Net Cost Savings to Health Care Systems: Results from a Comprehensive European Decision Analytic Model".

Fildes et al. have compared our data and results with those from the Swedish Obese Subjects study [1]. Our results are to $98 \%$ based on gastric bypass surgery, the dominating procedure in Sweden and in Europe. The SOS study comprises only $13 \%$ gastric bypass patients, with the majority of patients operated with purely restrictive operations, $68 \%$ vertical banded gastroplasty (VBG) and $19 \%$ gastric banding. It has been clearly shown that VBG is accompanied by high costs, since up to $89 \%$ of patients need a conversion [2]. In our analysis, reduction of use of gastric bypass with corresponding increase in other types of surgeries led to reduction of costsaving effect.

The comparison with the report by Weiner et al. [3] is not quite appropriate either. That study has observed costs over a 6-year period for a group of bariatric surgery patients, most of whom had had a gastric bypass, laparoscopic (38.3\%) or open (35.4\%). However, the control group was chosen because

Oleg Borisenko

oleg.borisenko@synergus.com

1 Synergus AB, Svardvagen 19, 18233 Danderyd, Sweden

2 Aarhus University, Aarhus, Denmark

3 Aleris Hamlet Hospital Aarhus, Aarhus, Denmark

4 Imperial College London, London, UK

5 Aleris Obesity, Lund, Sweden

6 Lund University, Lund, Sweden patients had diseases that were obesity-related. The study reported a significant loss to follow-up over 6 years. Total costs were higher in years 2 and 3 for the surgery group, but these differences disappeared with time. Weiner et al. did not have data beyond year 6 . Since we report that the economic benefit of surgery to health care system appears after 17 years, we believe the results from the Weiner's study do not contradict our conclusion.

Limited duration of follow-up is an obvious and most common limitation of published empirical research of economic consequences of bariatric surgery. Due to the initial high cost of surgery, cumulative cost of surgical treatment has shown to be higher compared with cumulative cost of alternative conservative management in a short- and mid-term time horizon. To overcome this limitation and to incorporate long-term consequence of treatments, we have employed lifetime horizon in our model.

Fildes et al. had commented on omitting some of obesityrelated costs in our analysis, which can contribute to costsaving effect. We have considered the costs, related to surgery, surgical complications, treatment of diabetes and cardiovascular disorders. These costs constitute about $80 \%$ of total obesity-related cost as demonstrated in earlier [4-6] studies, although was reported to be lower in more recent studies [7]. Swedish cost-of-illness studies in obesity have also focused only on diabetes and cardiovascular outcomes [8]. In our opinion, the most important costs are included into analysis. Nevertheless, we agree that that with evolvement of treatments (e.g. for cancer), the relative weight of these cost items may be greater over the time.

We agree with the comment that validation of the model with studies of bariatric surgery is of high value. In our analysis, incidence and remission of diabetes were validated with the Scandinavian Obesity Surgery Registry, and the number of cardiovascular outcomes and mortality was validated with the 
Action for Health in Diabetes (AHEAD) study of lifestyle interventions, which included 5145 overweight or obese patients with type 2 diabetes (start BMI for validation was $35.9 \mathrm{~kg} / \mathrm{m}^{2}$ ) [9]. It is acknowledged that there would always be not enough validation attempts, as clinical data evolve over time. We agree that additional specific validation exercises would be of benefit.

In their comment, Fildes et al. focused on the overall conclusion from the analysis, although important sub-group differences in economic outcomes were shown. Thus, costsaving effect was reported in the base-case analysis (with characteristics of the cohort, mimicking characteristics of real surgical candidates in Sweden) and single-cohort analysis of diabetic patients and severe to super obese non-diabetic patients. But in single-cohort analysis, we did not observe cost savings in patients with moderate and severe obesity and no diabetes at baseline.

Bariatric surgery is a good example of a rapidly evolving area with changes of surgical techniques, dramatic reduction in the use of open surgical approach and improved safety. Empirical economic data, although being the source of information of the greatest validity, are always available with delay, usually have limited follow-up and often do not correspond to the current treatment practice. In this case, decision analytic modelling has an inevitable complementary role to inform clinical and policy decision-making, acknowledging its simplification over reality. Omitting data generated by comprehensive modelling would lead to underestimation of true economic value of modern bariatric surgery.

Conflict of Interest Dr. Borisenko, Mr. Adam, Ms. Zhang and Ms. Colpan are employees of Synergus $\mathrm{AB}$, which received grant from Covidien Inc., during the conduct of the study. Dr. Hedenbro reports lecturing fees from Johnson \& Johnson and lecturing fees and research funding from Covidien Inc., all outside the submitted work. Drs. FunchJensen and Ahmed have nothing to disclose.

Open Access This article is distributed under the terms of the Creative Commons Attribution 4.0 International License (http:// creativecommons.org/licenses/by/4.0/), which permits unrestricted use, distribution, and reproduction in any medium, provided you give appropriate credit to the original author(s) and the source, provide a link to the Creative Commons license, and indicate if changes were made.

\section{References}

1. Neovius M, Narbro K, Keating C, et al. Health care use during 20 years following bariatric surgery. JAMA. 2012;308(11):1132-41.

2. Werling M, Fändriks L, Björklund $P$, et al. Long-term results of a randomized clinical trial comparing roux-en-Y gastric bypass with vertical banded gastroplasty. Br J Surg. 2013;100(2):222-30.

3. Weiner JP, Goodwin SM, Chang HY, et al. Impact of bariatric surgery on health care costs of obese persons: a 6-year follow-up of surgical and comparison cohorts using health plan data. JAMA Surg. 2013;148(6):555-62.

4. Wolf AM, Colditz GA. Current estimates of the economic cost of obesity in the United States. Obes Res. 1998;6(2):97-106.

5. Birmingham CL, Muller JL, Palepu A, et al. The cost of obesity in Canada. CMAJ. 1999;160(4):483-8.

6. Lévy E, Lévy P, Le Pen C, et al. The economic cost of obesity: the French situation. Int J Obes Relat Metab Disord. 1995;19(11):788-92.

7. Moffatt E, Shack LG, Petz GJ, et al. The cost of obesity and overweight in 2005: a case study of Alberta Canada. Can J Public Health. 2011;102(2):144-8.

8. Odegaard K, Borg S, Persson U, et al. The Swedish cost burden of overweight and obesity-evaluated with the PAR approach and a statistical modelling approach. Int J Pediatr Obes. 2008;3 Suppl 1: $51-7$.

9. Wing RR, Bolin P, Brancati FL, et al. Cardiovascular effects of intensive lifestyle intervention in type 2 diabetes. N Engl J Med. 2013;369(2):145-54. 